\title{
Article \\ Headache Because of Problems with Teeth, Mouth, Jaws, or Dentures in Chronic Temporomandibular Disorder Patients: A Case-Control Study
}

\author{
Tadej Ostrc ${ }^{1,2, *(\mathbb{D})}$, Sabina Frankovič ${ }^{3}$, Zvezdan Pirtošek ${ }^{4,5}$ and Ksenija Rener-Sitar ${ }^{1,2}$ (]) \\ 1 Department of Dental Prosthetics, Dental Division, Faculty of Medicine, University of Ljubljana, \\ 1000 Ljubljana, Slovenia; ksenija.rener@mf.uni-lj.si \\ 2 Department of Prosthetic Dentistry, Division of Stomatology, University Medical Center Ljubljana, \\ 1000 Ljubljana, Slovenia \\ 3 Mental Health Dispensary, Community Health Centre Kranj, 4000 Kranj, Slovenia; \\ sabina.frankovic@gmail.com \\ 4 Department of Neurology, University Medical Center Ljubljana, 1000 Ljubljana, Slovenia; \\ zvezdan.pirtosek@kclj.si \\ 5 Department of Neurology, Faculty of Medicine, University of Ljubljana, 1000 Ljubljana, Slovenia \\ * Correspondence: tadej.ostrc@mf.uni-lj.si; Tel.: +386-1-522-42-72
}

check for updates

Citation: Ostrc, T.; Frankovič, S.; Pirtošek, Z.; Rener-Sitar, K. Headache Because of Problems with Teeth, Mouth, Jaws, or Dentures in Chronic Temporomandibular Disorder Patients: A Case-Control Study. Int. J. Environ. Res. Public Health 2022, 19, 3052. https://doi.org/10.3390/ ijerph19053052

Academic Editor: Paul B. Tchounwou

Received: 28 January 2022

Accepted: 3 March 2022

Published: 5 March 2022

Publisher's Note: MDPI stays neutral with regard to jurisdictional claims in published maps and institutional affiliations.

Copyright: (C) 2022 by the authors. Licensee MDPI, Basel, Switzerland. This article is an open access article distributed under the terms and conditions of the Creative Commons Attribution (CC BY) license (https:// creativecommons.org/licenses/by/ $4.0 /)$.

\begin{abstract}
This study aimed to characterize self-reported headaches because of problems with the teeth, mouth, jaws, or dentures (HATMJD) in chronic patients with temporomandibular disorders (TMDs) in order to compare their results with those of TMD patients without such headaches and to investigate the associations of HATMJD with depression, anxiety, physical symptoms, oral behaviors, and sleep quality. We conducted a case-control study on consecutive chronic TMD patients referred to the University Medical Center of Ljubljana, Slovenia. A self-reported HATMJD was extracted from item \#12 in the 49-item version of the Oral Health Impact Profile questionnaire. Axis II instruments of the Diagnostic Criteria for TMD (i.e., for screening of depression, anxiety, specific comorbid functional disorders, and oral behaviors) and the Pittsburgh Sleep Quality Index were used in this study. In total, 177 TMD patients (77.4\% women; mean age: 36.3 years) participated in this study; $109(61.6 \%)$ patients were classified as TMD patients with HATMJD. TMD patients with at least mild depressive and anxiety symptoms, with at least low somatic symptom severity, and a high number of parafunctional behaviors had more HATMJD. Parafunctional behavior and sleep quality were the most prominent predictive factors of the occurrence of HATMJD. TMD patients with HATMJD have more psychosocial dysfunction, a higher frequency of oral behaviors, and poorer sleep quality than TMD patients without such headaches.
\end{abstract}

Keywords: temporomandibular disorders; headache; depression; anxiety; somatization; sleep quality

\section{Introduction}

Many clinical studies have confirmed associations between temporomandibular disorders (TMD) and tension-type headaches or migraine [1-4]. TMD and headaches may have common pathogenesis, causation, or common disruptive factors $[3,5,6]$. Furthermore, musculoskeletal pain in the orofacial area is more common in people with headaches [7]. In addition, TMD in patients with severe headaches have more significant pain intensity [7-12].

It is generally accepted that TMDs have a multifactorial etiology and that psychosocial components, including oral behaviors and trauma, contribute to the development, exacerbation, and progression to chronic TMD [13,14]. However, the role of psychological factors in the development of TMD disorders is still not entirely clear [15]. Some studies have concluded that patients with chewing muscle pain are more prone to stress and depression $[16,17]$. Stress and anxiety are the well-known psychological factors that correlate with TMD because stress can cause muscle hyperactivity, resulting in symptoms of TMD [15,18]. 
Psychological distress (e.g., anxiety, depression, and specific comorbid functional disorders) increases the symptom burden and functional impairment in several chronic medical conditions (e.g., headaches) [19-22]. For example, approximately half of acute TMD patients and $10 \%$ of headache sufferers have comorbid anxiety disorders, indicating that anxiety may be an early feature in both patient populations [20].

Psychopathology may also discriminate between myogenous and arthrogenous groups of TMD patients [14]. According to Gatchel et al., depression is more common among individuals with chronic tension-type headaches than with TMD [21]. Furthermore, the study by van der Meer et al. has shown that the associations between self-reported headache and painful/function-related TMD were confounded by the presence of somatic symptom complaints [22].

Oral parafunctions include sleep and awake bruxism, lip biting, thumb sucking, and any other oral habit not associated with mastication, deglutition, and speech [23]. The most common oral parafunctional activity is sleep bruxism, with a prevalence of up to $90 \%$ in the general population [24]. The relationship between oral parafunctions and TMD signs and symptoms has been reported in more studies $[25,26]$. An association between headache and bruxism has also been extensively explored [27]. Glaros et al. assessed oral parafunctions in headache and non-headache control groups and reported that headache patients had significantly more frequent and intense tooth contacts, more masticatory muscle tension, and more stress than non-pain controls [28]. Another study by Glaros et al. showed that subjects with headaches were significantly more likely to receive a diagnosis of masticatory myofascial pain than those in the non-headache control [29].

The substantial relationship of myofascial pain on sleep quality is documented in the study, which assessed differences in sleep quality between patients with chronic daily headaches and patients with TMD [30]. It was found that sleep quality was significantly worse in TMD patients with myofascial pain than patients with chronic daily headaches and intracapsular pain [30]. Therefore, assessing sleep quality must also be considered for TMD patients who are chronically distressed by their condition and is highly recommended in patients with dysfunctional TMD pain [31]. Sleep disturbances and headache disorders have similar pathogenic mechanisms; therefore, tension-type headaches, migraines, and sleep disturbances often occur together $[32,33]$. Insomnia is a known risk factor for headaches, especially tension-type headaches and migraines [34]. Sleep disruption is a common problem among subjects reporting headaches, and it is reported that sleep quality exhibits a complex interaction in individuals with chronic tension-type headaches [35]. Other studies have reported that poor sleep quality is not correlated with migraine [36]. A recent study has shown that patients with more severe insomnia present more severe depressive and anxiety symptoms [37].

The diagnosis of "headache attributed to temporomandibular disorder" (HATMD) was first introduced as a secondary headache in the second edition of International Classification of Headache Disorders (ICHD-II) under the code 11.7 by the Headache Classification Subcommittee of the International Headache in 2004 and further defined in the third version (i.e., ICHD-III) in 2018 [38]. The HATMD diagnosis is defined as headache and facial pain due to problems in the temporomandibular joints (TMJs), masticatory muscles, and/or associated structures after all primary headaches are excluded [38]. Similarly, the "Diagnostic Criteria for Temporomandibular Disorders" (DC/TMD) also include HATMD, which further implies that orofacial musculoskeletal pain and/or TMD are associated with headache [39]. The temporal region, preauricular area, and/or masseter muscle are commonly tender in HATMD [39]. Unilateral or bilateral HATMD follows the pattern of affected ipsilateral or bilateral temporomandibular regions, respectively [38].

Primary headaches (e.g., migraine and tension-type headaches) contribute to TMD problems [27]. Conversely, muscle-related TMD are highly associated with the presence of migraine [40,41]. Although many studies explored associations between psychological distress, oral behaviors and/or sleep quality and TMD, or the same factors in patients with HATMD, none have explored the extent of associations of self-reported headache 
because of problems with teeth, mouth, jaws, or dentures (HATMJD), with psychological distress, oral behaviors, and sleep quality in TMD patients, which is a clinically relevant question. Therefore, this study aimed to characterize self-reported HATMJD in chronic TMD patients, to compare their results with those of TMD patients without such headaches, and investigate the associations of HATMJD with depression, anxiety, physical symptoms, oral behaviors, and sleep quality.

\section{Materials and Methods}

\subsection{Ethical Approval}

This research was approved by the Faculty of Arts' Ethics Committee, University of Ljubljana, Slovenia, on 6 June 2016, and by the National Medical Ethics Committee of the Republic of Slovenia (KME 124/05/16). All participants signed a statement regarding informed consent before beginning the study.

\subsection{Observed Population}

This case-control study was performed on consecutive adult chronic TMD patients referred for TMD management to the Clinic for Orofacial Pain and Dental Sleep Medicine within the Department for Prosthetic Dentistry (University Dental Clinics, University Medical Center Ljubljana, Slovenia) between March 2016 and February 2021. Exclusion criteria included being aged less than 18, the presence of orofacial pain disorders other than TMD, or systemic rheumatic diseases. The diagnosis of TMD was based on history and physical examination findings according to DC/TMD protocol by a board-certified specialist for orofacial pain. The baseline data prior to treatment were analyzed for this study.

\subsection{Study Instruments}

We collected the data about demographics, such as gender, age, marital status, and level of education. The presence of the self-reported HATMJD was extracted from the Slovenian version of the psychometrically validated 49-item Oral Health Impact Profile questionnaire (OHIP), specifically from item \#12 ("Have you had headaches because of problems with your teeth, mouth, jaws or dentures?") [42]. Responses regarding the HATMJD were rated on the following scale: $0=$ "Never", $1=$ "Hardly ever", 2 = "Occasionally", $3=$ "Fairly often", and $4=$ "Very often" [43].

Additionally, Axis II instruments of the Diagnostic Criteria for TMD (i.e., for screening of depression, anxiety, specific comorbid functional disorders, and oral behaviors) and the Pittsburgh Sleep Quality Index were used in this study. All these questionnaires were translated into the Slovenian language and psychometrically validated in the Slovenian TMD population in the previous study [44].

Depression. PHQ-9 was introduced in 2001 and is designed to screen and measure the severity of depression [44]. Patients are asked how often they were bothered by problems over the last two weeks. Answers are formulated on a scale from 0 (not at all) to 3 (nearly every day). The total score for the nine items ranges from 0 to 27 . Higher scores represent a higher level of depression. Scores of 5, 10, 15, and 20 represent the cut-off points for mild, moderate, moderately severe, and severe depressive symptoms, respectively. Cronbach's alpha for measurement of internal consistency for the Slovenian version was 0.75 when assessed in Slovenian TMD patients [45].

Anxiety. GAD-7 was introduced in 2006 as a self-reported questionnaire to screen and measure generalized anxiety disorder severity [46]. Patients are asked how often they were bothered by problems over the last two weeks. Answers are formulated on a scale from 0 (not at all) to 3 (nearly every day). The total score for the seven items ranges from 0 to 21 . A higher score represents a higher level of anxiety. Scores of 5, 10, and 15 are the cut-off points for mild, moderate, and severe anxiety, respectively. The internal consistency of this questionnaire for the Slovenian version is high when assessed in Slovenian TMD patients, with a Cronbach's alpha of 0.88 [45]. 
Physical symptoms. PHQ-15 was introduced in 2002 to inquire about 15 somatic symptoms (i.e., specific comorbid functional disorders) that account for more than $90 \%$ of the physical complaints [47]. Subjects are asked to rate the severity of each symptom as 0 (not bothered at all), 1 (bothered a little), or 2 (bothered a lot) over the previous week. The summary score ranges from 0 to 30 . A higher score indicates a higher level of somatic symptom severity. Scores of 5, 10, and 15 are the cut-off points for low, medium, and high somatic symptom severity, respectively. The Cronbach's alpha for the Slovenian version is 0.69 when assessed in Slovenian TMD patients [45].

Parafunctions. The Oral Behavior Checklist (OBC) is a self-report scale for identifying and quantifying the frequency of jaw overuse behaviors (e.g., grinding, clenching teeth together, tightening and tensing the masticatory muscles, abnormal jaw posture, intense pressing of the tongue against the teeth, excessive talking, yawning) [48]. For each item, the subject is asked to report the frequency of the occurrence of specific oral behaviors over the past month, using the response options "none of the time", "a little of the time", "some of the time," "most of the time", and "all of the time" on a scale from 0 to 4 . The summary score ranges from 0 to 84 . A higher score indicates a higher number of parafunctional activities. A score of 0 indicates no parafunctional activity. Scores up to 24 indicate a low frequency of jaw overuse behaviors, whereas scores of 25 or more indicate a high frequency of jaw overuse behaviors.

Sleep quality. The Pittsburgh Sleep Quality Index (PSQI) measures the self-reported quality and patterns of sleep in adults [31]. It differentiates "poor" from "good" sleep by measuring seven areas: subjective sleep quality, sleep latency, sleep duration, habitual sleep efficiency, sleep disturbances, use of sleeping medication, and daytime dysfunction over the last month. The patient self-rates these seven areas of sleep. The answers are based on a 0 to 3 scale, in which 3 reflects the negative extreme on the Likert scale. Summary scores range from 0 to 21, and scores higher than 5 indicate poor sleep quality. The Slovenian version of PSQI has a Cronbach's alpha reliability coefficient of 0.74 for its seven components [49].

\subsection{Data Collection}

All questionnaires were administered to our TMD patient cohort in electronic form before treatment. The questionnaires were presented in 1KA software [50], a free, opensource application for online surveys. In addition, each patient received a unique and anonymous code to register on the platform to complete the questionnaires at home.

For this study purpose, the TMD patients were subsequently divided according to the presence or absence of the self-reported HATMJD, based on item \#12 from the OHIP questionnaire results. If the TMD patients responded to item \#12 with "Never" or "Hardly ever", we interpreted this as the absence of HATMJD. In contrast, if they answered to this item as "Occasionally", "Fairly often", or "Very often", we interpreted this as the presence of HATMJD. Therefore, the TMD cohort was stratified into the HATMJD and non-HATMJD groups, which were our study and control groups, respectively.

\subsection{Data Analysis}

The variables analyzed as potential explanatory factors responsible for the variability of our observed outcome (i.e., the presence of HATMJD) were patient demographics, depression, anxiety, specific comorbid functional disorders, parafunctional behaviors, and sleep quality. Because measures for our constructs were continuous or ordinal in their original metric (e.g., the Likert-type scale), our TMD patients were grouped according to DC/TMD recommendations as follows: depression (no, mild, moderate, moderately severe, or severe depressive symptoms), anxiety (no, mild, moderate and severe anxiety), specific comorbid functional disorders (no, low, medium or high somatic symptom severity), and parafunctional behaviors (none, low or high) [51]. Sleep quality was grouped as good or poor (i.e., a PSQI score greater than five was considered poor sleep quality). According to Colarusso, the young adult population was defined as below 40 years of age [52]. 
The effect sizes with 95\% confidence intervals (i.e., Cohen's d) demonstrated the standardized differences between the two groups. Standard guidelines for interpreting effect size suggest that 0.2 is considered small, 0.5 medium, and 0.8 a large effect [53]. The associations between our observed outcome (i.e., the presence of HATMJD, and explanatory factors, e.g., depression, anxiety, specific comorbid functional disorders, parafunctional behaviors, and sleep quality) were first assessed with simple logistic regression analysis. Subsequently, the explanatory factors, which proved statistically significant, were entered in multiple logistic regression analysis, with stepwise forward variable selection (likelihood ratio) with HATMJD presence as the outcome to identify the significant associations between the predictors, which were independent variables and our observed outcome, being dependent variable, which is the presence of HATMJD.

The model's predictive power was evaluated using the Omnibus Test of Model, and the Hosmer-Lemeshow test was used to examine the goodness of the model's fit [54]. Nagelkerke's R-square was generated to express the proportion of variance explained by the model [55]. The odds ratio (OR) and 95\% confidence intervals (95\% CI) were calculated in simple logistic regression and multiple logistic regression models. Statistical significance was set at the level of $p<0.05$. Statistical analysis was performed in SPSS for Windows software, version 25.0 (IBM Corporation, Armonk, NY, USA).

\section{Results}

One hundred and seventy-seven adult TMD patients (137 women; patient cohort mean age: 36.3 years) participated in this study. All participants answered all items in the previously described questionnaires. Of all included TMD patients, $109(61.6 \%)$ patients were classified as TMD patients with HATMJD, whereas $68(38.4 \%)$ TMD patients reported no presence of HATMJD. The demographic and clinical characteristics of the studied TMD patient cohort and its subgroups (i.e., the study group of TMD patients with a HATMJD) and the second subgroup (i.e., the control group of TMD patients without self-reported HATMJD) are presented in Table 1.

Table 1. Descriptive statistics of studied TMD patient cohort and its subgroups (i.e., the study group of TMD patients with a self-reported headache because of problems with teeth, mouth, jaws, or dentures (HATMJD)) and the second subgroup (i.e., the control group of TMD patients without self-reported HATMJD).

\begin{tabular}{|c|c|c|c|c|}
\hline \multirow[b]{2}{*}{ Patients' Characteristics } & \multicolumn{3}{|c|}{ Mean \pm SD or $n(\%)$} & \multirow[b]{2}{*}{$p$-Value } \\
\hline & All TMD pts & $\begin{array}{l}\text { Study Group } \\
\text { TMD pts w } \\
\text { HATMJD }\end{array}$ & $\begin{array}{l}\text { Control Group } \\
\text { TMD pts w/o } \\
\text { HATMJD }\end{array}$ & \\
\hline No. of patients & $177(100)$ & 109 (61.6) & $68(38.4)$ & \\
\hline \multicolumn{5}{|l|}{ Sociodemographic } \\
\hline Female gender & $137(77.4)$ & $92(84.4)$ & $45(66.2)$ & 0.005 * \\
\hline Age & $36.3 \pm 13.7$ & $35.07 \pm 12.2$ & $38.29 \pm 15.7$ & 0.153 \\
\hline Marital status & & & & 0.637 \\
\hline Single/widowed/divorced & $69(39.0)$ & $41(37.6)$ & $28(41.2)$ & \\
\hline Married/in relationship & $108(61.0)$ & $68(62.4)$ & $40(58.8)$ & \\
\hline Education & & & & 0.626 \\
\hline No college & $87(49.1)$ & $52(47.7)$ & $35(51.5)$ & \\
\hline One year of college or higher & $90(50.9)$ & $57(52.3)$ & $33(48.5)$ & \\
\hline $\begin{array}{c}\text { Depression (PHQ-9 } \\
\text { summary score) }\end{array}$ & $5.8 \pm 5.0$ & $6.6 \pm 5.3$ & $4.6 \pm 4.1$ & $0.011 *$ \\
\hline No depression & $86(48.6)$ & $47(43.1)$ & $39(57.4)$ & \\
\hline Mild depression & $62(35.0)$ & $41(37.6)$ & $21(30.9)$ & \\
\hline Moderate depression & $18(10.2)$ & $13(11.9)$ & $5(7.4)$ & \\
\hline
\end{tabular}


Table 1. Cont.

\begin{tabular}{|c|c|c|c|c|}
\hline \multirow[b]{2}{*}{ Patients' Characteristics } & \multicolumn{3}{|c|}{ Mean \pm SD or $n(\%)$} & \multirow[b]{2}{*}{$p$-Value } \\
\hline & All TMD pts & $\begin{array}{l}\text { Study Group } \\
\text { TMD pts w } \\
\text { HATMJD }\end{array}$ & $\begin{array}{l}\text { Control Group } \\
\text { TMD pts w/o } \\
\text { HATMJD }\end{array}$ & \\
\hline $\begin{array}{c}\text { Moderately severe } \\
\text { depression }\end{array}$ & $7(3.9)$ & $4(3.7)$ & $3(4.4)$ & \\
\hline Severe depression & $4(2.3)$ & $4(3.7)$ & / & \\
\hline $\begin{array}{c}\text { Anxiety (GAD-7 summary } \\
\text { score) }\end{array}$ & $6.0 \pm 5.1$ & $6.7 \pm 5.2$ & $4.9 \pm 4.8$ & $0.020 *$ \\
\hline No anxiety & $80(45.2)$ & $43(39.4)$ & $37(54.4)$ & \\
\hline Mild anxiety & $62(35.0)$ & $42(38.5)$ & $20(29.4)$ & \\
\hline Moderate anxiety & $20(11.3)$ & $15(13.8)$ & $5(7.4)$ & \\
\hline Severe anxiety & $15(8.5)$ & $9(8.3)$ & $6(8.8)$ & \\
\hline $\begin{array}{c}\text { Physical Symptoms } \\
\text { (PHQ-15 summary score) }\end{array}$ & $7.4 \pm 4.4$ & $8.4 \pm 4.4$ & $6.0 \pm 4.1$ & $0.001 *$ \\
\hline No somatic symptoms & $47(26.5)$ & $20(18.3)$ & $27(39.7)$ & \\
\hline $\begin{array}{c}\text { Low somatic symptom } \\
\text { severity }\end{array}$ & $81(45.8)$ & $52(47.7)$ & $29(42.6)$ & \\
\hline $\begin{array}{c}\text { Medium somatic symptom } \\
\text { severity }\end{array}$ & $37(20.9)$ & $27(24.8)$ & $10(14.7)$ & \\
\hline $\begin{array}{l}\text { High somatic symptom } \\
\text { severity }\end{array}$ & $12(6.8)$ & $10(9.2)$ & $2(2.9)$ & \\
\hline $\begin{array}{l}\text { Oral Behaviors (OBC } \\
\text { summary score) }\end{array}$ & $23.9 \pm 8.6$ & $26.1 \pm 8.0$ & $20.2 \pm 8.5$ & $<0.001$ * \\
\hline None & / & / & / & \\
\hline Low & 105 (59.3) & $52(47.7)$ & $53(77.9)$ & \\
\hline High & $72(40.7)$ & $57(52.3)$ & $15(22.1)$ & \\
\hline $\begin{array}{l}\text { Sleep Quality (PSQI } \\
\text { summary score) }\end{array}$ & $6.0 \pm 3.6$ & $6.8 \pm 3.7$ & $4.6 \pm 2.8$ & $<0.001 *$ \\
\hline Good sleep quality & $80(45.2)$ & $38(34.9)$ & $42(61.8)$ & \\
\hline Poor sleep quality & $97(54.8)$ & $71(65.1)$ & $26(38.2)$ & \\
\hline
\end{tabular}

TMD: Temporomandibular disorders; PHQ-9: Patient Health Questionnaire-9; GAD-7: General Anxiety Disorder7; PHQ-15: Patient Health Questionnaire-15; OBC: Oral Behaviors Checklist; PSQI: Pittsburgh Sleep Quality Index. $* p<0.05$.

Table 2 displays the analysis of the effect sizes (i.e., influences of sociodemographic variables and selected DC/TMD Axis II measures on HATMJD) using standardized mean effect reported as Cohen's d.

Table 2. Comparing the influence of depression, anxiety, functional disorders, oral behaviors, and sleep quality on headache because of problems with teeth, mouth, jaws, or dentures in TMD patients using standardized mean effect reported as Cohen's d.

\begin{tabular}{|c|c|c|c|c|c|}
\hline \multirow{2}{*}{ Variable } & \multirow{2}{*}{ Group } & \multirow{2}{*}{$\mathbf{N}$} & \multirow{2}{*}{ Cohen's d } & \multicolumn{2}{|c|}{$95 \%$ CI for Cohen's d } \\
\hline & & & & Lower & Upper \\
\hline \multicolumn{6}{|l|}{ Sociodemographic } \\
\hline Gender & $\begin{array}{l}\text { Male TMD pts w HATMJD vs. } \\
\text { Female TMD pts w HATMJD }\end{array}$ & 40 vs. 137 & 0.62 & 0.26 & 0.97 \\
\hline Age & $<40$ y vs. $\geq 40 y$ & 120 vs. 57 & 0.07 & -0.24 & 0.39 \\
\hline Marital status & $\begin{array}{l}\text { Single/widowed/divorced } \\
\text { vs. Married/in relationship }\end{array}$ & 69 vs. 108 & $<0.00$ & -0.03 & 0.03 \\
\hline Education & $\begin{array}{l}\text { No college vs. } \geq 1 \text { years of } \\
\text { college }\end{array}$ & 87 vs. 90 & 0.15 & -0.14 & 0.45 \\
\hline
\end{tabular}


Table 2. Cont.

\begin{tabular}{|c|c|c|c|c|c|}
\hline \multirow{2}{*}{ Variable } & \multirow{2}{*}{ Group } & \multirow{2}{*}{$\mathbf{N}$} & \multirow{2}{*}{ Cohen's d } & \multicolumn{2}{|c|}{ 95\% CI for Cohen's d } \\
\hline & & & & Lower & Upper \\
\hline \multicolumn{6}{|l|}{$\begin{array}{c}\text { DC/TMD Axis II } \\
\text { diagnoses }\end{array}$} \\
\hline Depression & No vs. present & 86 vs. 91 & 0.35 & 0.05 & 0.64 \\
\hline Physical symptoms & No vs. present & 47 vs. 130 & 0.62 & 0.28 & 0.96 \\
\hline Anxiety & No vs. present & 80 vs. 97 & 0.45 & 0.15 & 0.75 \\
\hline $\begin{array}{c}\text { Parafunctional } \\
\text { behaviors }\end{array}$ & None to low vs. high & 105 vs. 72 & 0.70 & 0.39 & 1.01 \\
\hline \multicolumn{6}{|l|}{ Other constructs } \\
\hline Sleep quality & Good vs. poor & 80 vs. 97 & 0.64 & 0.34 & 0.94 \\
\hline
\end{tabular}

TMD: Temporomandibular disorders; w HATMJD: with a headache because of problems with teeth, mouth, jaws, or dentures; DC/TMD: Diagnostic Criteria for Temporomandibular Disorders.

The results of the simple logistic regression analysis are presented in Table 3.

Table 3. Simple logistic regression analysis predicting the presence of self-reported headache because of problems with teeth, mouth, jaws, or dentures in TMD patients.

\begin{tabular}{ccccc}
\hline Predicting Factor & Odds Ratio & $p$-Value & \multicolumn{2}{c}{$\mathbf{9 5 \% \text { CI for Odds Ratio }}$} \\
\cline { 4 - 5 } & & & Lower & Upper \\
\hline Female gender & 2.77 & $0.006^{*}$ & 1.34 & 5.69 \\
Age & 0.98 & 0.130 & 0.96 & 1.00 \\
Marital status & 0.64 & 0.637 & 0.46 & 1.60 \\
Higher level of education & 1.16 & 0.626 & 0.63 & 2.13 \\
Depression & 1.10 & $0.015^{*}$ & 1.02 & 1.18 \\
Anxiety & 1.08 & $0.023^{*}$ & 1.01 & 1.15 \\
Physical symptoms & 1.15 & $0.001^{*}$ & 1.06 & 1.25 \\
Oral behaviors & 1.20 & $<0.001^{*}$ & 1.09 & 1.34 \\
Sleep quality & 1.24 & $<0.001^{*}$ & 1.11 & 1.39 \\
\hline *p & & & &
\end{tabular}

*p<0.05.

The complete five-step multiple logistic regression analysis is shown in Table 4.

Table 4. Multiple logistic regression analysis, with stepwise forward variable selection method with a headache because of problems with teeth, mouth, jaws, or dentures presence as the outcome.

\begin{tabular}{|c|c|c|c|c|c|c|}
\hline \multirow[t]{2}{*}{ Step \# } & \multirow{2}{*}{$\begin{array}{l}\text { Predicting } \\
\text { Variable }\end{array}$} & \multirow[t]{2}{*}{$\beta$ Coefficient } & \multirow[t]{2}{*}{$p$-Value } & \multirow{2}{*}{$\begin{array}{l}\text { Odds } \\
\text { Ratio }\end{array}$} & \multicolumn{2}{|c|}{$\begin{array}{c}\text { 95\% CI for Odds } \\
\text { Ratio }\end{array}$} \\
\hline & & & & & Lower & Upper \\
\hline \multirow{4}{*}{ Step 1} & Age & -0.010 & 0.415 & 0.990 & 0.967 & 1.014 \\
\hline & Female gender & 0.932 & 0.018 * & 2.538 & 1.174 & 5.486 \\
\hline & OBC & 0.086 & $<0.001 *$ & 1.090 & 1.043 & 1.138 \\
\hline & Constant & -1.857 & 0.018 & 0.156 & & \\
\hline \multirow{5}{*}{ Step 2} & Age & -0.015 & 0.243 & 0.985 & 0.961 & 1.010 \\
\hline & Female gender & 0.992 & 0.014 * & 2.696 & 1.218 & 5.964 \\
\hline & OBC & 0.056 & 0.026 * & 1.058 & 1.007 & 1.111 \\
\hline & PSQI & 0.156 & 0.015 * & 1.169 & 1.031 & 1.325 \\
\hline & Constant & -1.857 & 0.017 & 0.148 & & \\
\hline \multirow{6}{*}{ Step 3} & Age & -0.015 & 0.243 & 0.985 & 0.961 & 1.010 \\
\hline & Female gender & 0.963 & $0.022 *$ & 2.619 & 1.149 & 5.973 \\
\hline & OBC & 0.055 & 0.028 * & 1.057 & 1.006 & 1.111 \\
\hline & PSQI & 0.147 & 0.045 * & 1.158 & 1.003 & 1.338 \\
\hline & PHQ-15 & 0.014 & 0.804 & 1.014 & 0.908 & 1.132 \\
\hline & Constant & -1.857 & 0.017 & 0.147 & & \\
\hline
\end{tabular}


Table 4. Cont.

\begin{tabular}{|c|c|c|c|c|c|c|}
\hline \multirow[t]{2}{*}{ Step \# } & \multirow{2}{*}{$\begin{array}{l}\text { Predicting } \\
\text { Variable }\end{array}$} & \multirow[t]{2}{*}{$\beta$ Coefficient } & \multirow[t]{2}{*}{$p$-Value } & \multirow{2}{*}{$\begin{array}{l}\text { Odds } \\
\text { Ratio }\end{array}$} & \multicolumn{2}{|c|}{$\begin{array}{c}95 \% \text { CI for Odds } \\
\text { Ratio }\end{array}$} \\
\hline & & & & & Lower & Upper \\
\hline \multirow{7}{*}{ Step 4} & Age & -0.018 & 0.170 & 0.982 & 0.958 & 1.008 \\
\hline & Female gender & 0.949 & 0.025 * & 2.583 & 1.129 & 5.910 \\
\hline & OBC & 0.059 & 0.021 * & 1.061 & 1.009 & 1.116 \\
\hline & PSQI & 0.188 & $0.021 *$ & 1.207 & 1.029 & 1.416 \\
\hline & PHQ-15 & 0.038 & 0.522 & 1.039 & 0.924 & 1.168 \\
\hline & PHQ-9 & -0.073 & 0.204 & 0.930 & 0.831 & 1.040 \\
\hline & Constant & -1.892 & 0.019 & 0.151 & & \\
\hline \multirow{8}{*}{ Step 5} & Age & -0.018 & 0.159 & 0.982 & 0.957 & 1.007 \\
\hline & Female gender & 0.969 & 0.023 * & 2.636 & 1.145 & 6.070 \\
\hline & OBC & 0.061 & 0.019 * & 1.063 & 1.010 & 1.118 \\
\hline & PSQI & 0.188 & 0.021 * & 1.207 & 1.029 & 1.415 \\
\hline & PHQ-15 & 0.038 & 0.528 & 1.039 & 0.923 & 1.168 \\
\hline & PHQ-9 & -0.048 & 0.549 & 0.953 & 0.815 & 1.115 \\
\hline & GAD-7 & -0.028 & 0.659 & 0.972 & 0.857 & 1.102 \\
\hline & Constant & -1.892 & 0.019 & 0.151 & & \\
\hline
\end{tabular}

PHQ-9: Patient Health Questionnaire-9; GAD-7: General Anxiety Disorder-7; PHQ-15: Patient Health Questionnaire-15; OBC: Oral Behaviors Checklist; PSQI: Pittsburgh Sleep Quality Index. ${ }^{*} p<0.05$. The goodness of fit: $\chi^{2}<0.05$.

The final model had a statistically significant predictive power $\left(\chi^{2}=32.399, p<0.001\right.$; Hosmer-Lemeshow test for goodness of fit: $\chi^{2}=10.206, p=0.251$, and overall, correctly classified $70.6 \%$ of the TMD patients. The final model explained $22.7 \%$ of the variance for the presence of HATMJD (Nagelkerke's R square was 0.227).

\section{Discussion}

Our study showed that TMD patients with a self-reported HATMJD are mainly women, with greater chance of psychological dysfunction, higher frequency of oral behaviors, and poorer sleep quality than TMD patients without such headaches. Statistically significant factors from simple logistic regression analysis (i.e., gender, depression, anxiety, specific comorbid functional disorders, oral behaviors, and sleep quality) were included in multiple logistic regression analyses to identify the significant associations between the predictors (independent variables) and the outcome (dependent variable: the presence of a HATMJD). In other words, we aimed to create the best regression model from our predictor variables that most accurately explain the associations between our predictor variables and the presence of HATMJD.

Our TMD patients with a high frequency of parafunctional activities had a 1.2-times higher chance of developing the self-reported HATMJD. Furthermore, in female TMD patients, the perceived HATMJD was 2.6-times more likely to occur than in male TMD patients. The female gender was the most prominent statistically significant sociodemographic characteristic in TMD patients with HATMJD. These findings are consistent with previous studies that investigated the co-morbidity of TMD and headaches $[7,22,56,57]$. Therefore, the self-reported HATMJD rate was statistically more frequent in female TMD patients than in male ones, and, when expressed as the effect size, it was medium. There was no statistically significant difference between the HATMJD group versus the non-HATMJD group for marital status and education. Additionally, the high number of parafunctional behaviors correlated statistically significantly with the presence of HATMJD, with an effect size close to large. The HATMJD presence was statistically higher in people with poorer sleep quality than in people with good sleep quality, and when expressed as effect size, it was medium.

Medium to high somatic symptom severity was reported in 34\%, moderate to severe anxiety in $19.8 \%$, and moderate to severe depression in $19.3 \%$ of TMD patients, who re- 
ported HATMJD in our study. The association between psychological distress (including depression, specific comorbid functional disorders, and anxiety) and TMD pain was investigated in some previous studies [58,59]. In the systematic review from De La Torre Canales et al., in which they included 14 studies investigating psychosocial impairment in TMD patients, the prevalence of medium to high somatic symptom severity varied from $28.5 \%$ to $76.6 \%$ and for moderate to severe depression from $21.4 \%$ to $60.1 \%$ [60]. It is important to emphasize that different study instruments for psychological distress were used in our study (i.e., PHQ-9 and PHQ-15) than in the study of De La Torre Canales et al., in which they utilized the instruments SCL-90-SOM and SCL-90-DEP, which could explain the differences in prevalence.

In the final model adjusted for age and gender, three variables were statistically significantly correlated with the presence of HATMJD in our TMD cohort. Female patients with poorer sleep quality and a higher frequency of parafunctional oral behaviors were more likely to have the presence of HATMJD. Therefore, oral behaviors and sleep quality when adjusted for gender are the statistically significant factors contributing to the perceived HATMJD. Our findings, therefore, are consistent with previous studies in patients with tension-type headaches and migraines [29,32], which showed that headache patients are significantly more likely to report oral parafunctional behaviors and that poor sleep quality is associated with higher severity of headaches. In addition, it is known that parafunctional oral behavior could effectively be addressed with early biobehavioral intervention [61,62] Such treatment could also potentially alleviate the self-reported headache in the TMD patient population [29].

Interestingly, in the recent study by Reiter et al., which retrospectively evaluated DC/TMD Axis I and Axis II data from 220 patients having pain-related TMD with HATMD, the mean depression scores measured with PHQ-9 were similar (6.88 \pm 4.69$)$ to our study $(6.6 \pm 5.3)$, mean anxiety scores, i.e., GAD-7 scores, were lower $(4.35 \pm 3.79)$ than in our study $(4.9 \pm 4.8)$, and somatic symptom severity, i.e., PHQ-15 scores, were also lower $(7.18 \pm 4.18)$ than in our study $(8.4 \pm 4.4)$ [63]. Although we included all TMD patients in our study and not only those with pain-related TMD, we still had higher anxiety and somatic symptom severity scores in comparison to the study by Reiter et al. [63], who included only patients with pain-related TMD and with comorbid HATMD.

The study of Tchivileva et al. reported a mean PSQI summary score of 6.0 in patients with comorbid tension-type headache and HATMD and 7.6 in patients with comorbid migraine and HATMD [64]. In contrast, in our study, the average sleep quality, reported as the PSQI score, was 6.8 for our TMD patients with HATMJD. Therefore, if we compare our patients' sleep quality with the sleep quality results by Tchivileva et al. [64], we see that our sleep quality results were exactly in between their sleep quality results for TMD patients with tension-type headaches and TMD patients with migraine. This finding is not surprising because HATMJD probably included at least some primary headaches.

To our knowledge, this is the first study to investigate the associations between all types of self-reported HATMJD in the TMD patient cohort and the psychosocial dysfunction, sleep quality, and parafunctional activities. Nevertheless, this study has limitations. We did not specify which TMD diagnosis is more associated with self-reported HATMJD. Our study aimed to focus on psychosocial predictors for HATMJD in TMD patients, and for this reason, we did the multiple logistic regression analysis on the complete TMD patient cohort. Additionally, the accuracy of self-perceived oral parafunctional behaviors is questionable when reported only using a questionnaire because most individuals with sleep bruxism are not aware of this oral behavior since it occurs during sleep.

\section{Conclusions}

TMD patients with at least mild depressive and anxiety symptoms, with at least low somatic symptom severity, and a high number of parafunctional behaviors, had more HATMJD. This is additional proof that the DC/TMD Axis II constructs are crucial outcome predictors for TMD treatment purposes and HATMJD in this patient population. 
Furthermore, this study indicates that besides HATMD, which is a secondary headache, other types of headaches, including the primary ones, are also associated with the DC/TMD Axis II constructs and sleep quality when present in TMD patients. Therefore, assessing psychological distress, oral behaviors, and sleep quality is highly recommended in TMD patients with comorbid headache(s). Future research could also focus on how and if various TMD treatment modalities also affect different types of headaches in TMD patients.

Author Contributions: Conceptualization, T.O., S.F., Z.P., and K.R.-S.; methodology, T.O., S.F., and K.R.-S.; software, S.F.; formal analysis, T.O., S.F., and K.R.-S.; investigation, T.O., S.F., and K.R.-S.; data curation, T.O. and S.F.; writing-original draft preparation, T.O., S.F., and K.R.-S.; writing-review and editing, T.O., S.F., Z.P., and K.R.-S.; funding acquisition, T.O., K.R.-S., and Z.P. All authors have read and agreed to the published version of the manuscript.

Funding: This research was funded by the Tertiary project UKC Ljubljana-Project number 20190168 with the title 'Prospektivna dvojno slepa randomizirana klinična raziskava zdravljenja glavobola zaradi temporomandibularnih motenj'.

Institutional Review Board Statement: This research was conducted in accordance with the latest revision of the Declaration of Helsinki and approved by the Faculty of Arts' Ethics Committee, University of Ljubljana, Slovenia, on 6 June 2016, and by the National Medical Ethics Committee of the Republic of Slovenia (KME 124/05/16).

Informed Consent Statement: Informed consent was obtained from all subjects involved in the study.

Data Availability Statement: The datasets generated during the current study are available from the corresponding author on reasonable request.

Conflicts of Interest: The authors declare no conflict of interest. The funders had no role in the design of the study; in the collection, analyses, or interpretation of data; in the writing of the manuscript; or in the decision to publish the results.

\section{References}

1. Nixdorf, D.R.; Velly, A.M.; Alonso, A.A. Neurovascular pains: Implications of migraine for the oral and maxillofacial surgeon. Oral Maxillofac. Surg. Clin. N. Am. 2008, 20, 221-235. [CrossRef] [PubMed]

2. Bevilaqua Grossi, D.; Lipton, R.B.; Bigal, M.E. Temporomandibular disorders and migraine chronification. Curr. Pain Headache Rep. 2009, 13, 314-318. [CrossRef] [PubMed]

3. da Silva, A., Jr.; Costa, E.C.; Gomes, J.B.; Leite, F.M.; Gomez, R.S.; Vasconcelos, L.P.; Krymchantowski, A.; Moreira, P.; Teixeira, A.L. Chronic headache and co-morbidities: A two-phase, population-based, cross-sectional study. Headache 2010, 50, 1306-1312. [CrossRef]

4. Goncalves, D.A.; Camparis, C.M.; Franco, A.L.; Fernandes, G.; Speciali, J.G.; Bigal, M.E. How to investigate and treat: Migraine in patients with temporomandibular disorders. Curr. Pain Headache Rep. 2012, 16, 359-364. [CrossRef]

5. Goncalves, D.A.; Camparis, C.M.; Speciali, J.G.; Castanharo, S.M.; Ujikawa, L.T.; Lipton, R.B.; Bigal, M.E. Treatment of comorbid migraine and temporomandibular disorders: A factorial, double-blind, randomized, placebo-controlled study. J. Orofac. Pain 2013, 27, 325-335. [CrossRef] [PubMed]

6. Melo, C.E.; Oliveira, J.L.; Jesus, A.C.; Maia, M.L.; de Santana, J.C.; Andrade, L.S.; Quintans, J.D.; Quintans-Junior, L.J.; Conti, P.C.; Bonjardim, L.R. Temporomandibular disorders dysfunction in headache patients. Med. Oral Patol. Oral Cir. Bucal. 2012, 17, e1042-e1046. [CrossRef]

7. Bingefors, K.; Isacson, D. Epidemiology, co-morbidity, and impact on health-related quality of life of self-reported headache and musculoskeletal pain-A gender perspective. Eur. J. Pain 2004, 8, 435-450. [CrossRef]

8. Magnusson, T.; Carlsson, G.E. Recurrent headaches in relation to temporomandibular joint pain-dysfunction. Acta Odontol. 1978, 36, 333-338. [CrossRef]

9. Pettengill, C. A comparison of headache symptoms between two groups: A TMD group and a general dental practice group. Cranio 1999, 17, 64-69. [CrossRef]

10. Ballegaard, V.; Thede-Schmidt-Hansen, P.; Svensson, P.; Jensen, R. Are headache and temporomandibular disorders related? A blinded study. Cephalalgia 2008, 28, 832-841. [CrossRef]

11. Goncalves, D.A.; Camparis, C.M.; Speciali, J.G.; Franco, A.L.; Castanharo, S.M.; Bigal, M.E. Temporomandibular disorders are differentially associated with headache diagnoses: A controlled study. Clin. J. Pain 2011, 27, 611-615. [CrossRef] [PubMed]

12. Di Paolo, C.; D’Urso, A.; Papi, P.; Di Sabato, F.; Rosella, D.; Pompa, G.; Polimeni, A. Temporomandibular Disorders and Headache: A Retrospective Analysis of 1198 Patients. Pain Res. Manag. 2017, 2017, 3203027. [CrossRef] [PubMed]

13. Okeson, J.P. Management of Temporomandibular Disorders and Occlusion, 7th ed.; Elsevier Mosby: St. Louis, MO, USA, 2013. 
14. Di Paolo, C.; Falisi, G.; Panti, F.; Di Giacomo, P.; Rampello, A. "RA.DI.CA." Splint for the Management of the Mandibular Functional Limitation: A Retrospective Study on Patients with Anterior Disc Displacement without Reduction. Int. J. Environ. Res. Public Health 2020, 17, 9057. [CrossRef] [PubMed]

15. Ohrbach, R.; Michelotti, A. The Role of Stress in the Etiology of Oral Parafunction and Myofascial Pain. Oral Maxillofac. Surg. Clin. N. Am. 2018, 30, 369-379. [CrossRef]

16. Yap, A.U.; Tan, K.B.; Chua, E.K.; Tan, H.H. Depression and somatization in patients with temporomandibular disorders. J. Prosthet. Dent. 2002, 88, 479-484. [CrossRef]

17. Velly, A.M.; Gornitsky, M.; Philippe, P. Contributing factors to chronic myofascial pain: A case-control study. Pain 2003, 104, 491-499. [CrossRef]

18. Sekulić, S.; John, M.T.; Bekes, K.; Al-Harthy, M.H.; Michelotti, A.; Reissmann, D.R.; Nikolovska, J.; Sanivarapu, S.; Lawal, F.B.; List, T; et al. Frequency of Four-dimensional Oral Health Problems across Dental Fields-A Comparative Survey of Slovenian and International Dentists. Zdr. Varst. 2021, 60, 210-220. [CrossRef] [PubMed]

19. Salazar, A.; Berrocal, L.; Failde, I. Prevalence of Migraine in General Spanish Population; Factors Related and Use of Health Resources. Int. J. Environ. Res. Public Health 2021, 18, 1145. [CrossRef]

20. Jerjes, W.; Madland, G.; Feinmann, C.; Hopper, C.; Kumar, M.; Upile, T.; Kudari, M.; Newman, S. A psychological comparison of temporomandibular disorder and chronic daily headache: Are there targets for therapeutic interventions? Oral Surg. Oral Med. Oral Pathol. Oral Radiol. Endod. 2007, 103, 367-373. [CrossRef]

21. Gatchel, R.J.; Garofalo, J.P.; Ellis, E.; Holt, C. Major psychological disorders in acute and chronic TMD: An initial examination. J. Am. Dent. Assoc. 1996, 127, 1365-1370, 1372, 1374. [CrossRef]

22. van der Meer, H.A.; Speksnijder, C.M.; Engelbert, R.H.H.; Lobbezoo, F.; Nijhuis-van der Sanden, M.W.G.; Visscher, C.M. The Association Between Headaches and Temporomandibular Disorders is Confounded by Bruxism and Somatic Symptoms. Clin. J. Pain 2017, 33, 835-843. [CrossRef] [PubMed]

23. Mehta, N.R.; Scrivani, S.J.; Spierings, E.L.H. Dental and Facial Pain. In Practical Management of Pain; Benzon, H.T., Rathmell, J.P., Wu, C.L., Turk, D.C., Argoff, C.E., Hurley, R.W., Eds.; Mosby: Philadelphia, PA, USA, 2014; pp. 424-440.

24. Wruble, M.K.; Lumley, M.A.; McGlynn, F.D. Sleep-related bruxism and sleep variables: A critical review. J. Craniomandib. Disord. 1989, 3, 152-158. [PubMed]

25. Michelotti, A.; Cioffi, I.; Festa, P.; Scala, G.; Farella, M. Oral parafunctions as risk factors for diagnostic TMD subgroups. J. Oral Rehabil. 2010, 37, 157-162. [CrossRef] [PubMed]

26. Miyake, R.; Ohkubo, R.; Takehara, J.; Morita, M. Oral parafunctions and association with symptoms of temporomandibular disorders in Japanese university students. J. Oral Rehabil. 2004, 31, 518-523. [CrossRef]

27. Réus, J.C.; Polmann, H.; Mendes Souza, B.D.; Flores-Mir, C.; Trevisol Bittencourt, P.C.; Winocur, E.; Okeson, J.; De Luca Canto, G. Association Between Primary Headache and Bruxism: An Updated Systematic Review. J. Oral Facial Pain Headache 2021, 35, 129-138. [CrossRef] [PubMed]

28. Glaros, A.G.; Urban, D.; Locke, J. Headache and temporomandibular disorders: Evidence for diagnostic and behavioural overlap. Cephalalgia 2007, 27, 542-549. [CrossRef] [PubMed]

29. Glaros, A.G.; Hanson, A.H.; Ryen, C.C. Headache and Oral Parafunctional Behaviors. Appl. Psychophysiol. Biofeedback 2014, 39, 59-66. [CrossRef]

30. Vazquez-Delgado, E.; Schmidt, J.E.; Carlson, C.R.; De Leeuw, R.; Okeson, J.P. Psychological and sleep quality differences between chronic daily headache and temporomandibular disorders patients. Cephalalgia 2004, 24, 446-454. [CrossRef]

31. Rener-Sitar, K.; John, M.T.; Pusalavidyasagar, S.S.; Bandyopadhyay, D.; Schiffman, E.L. Sleep quality in temporomandibular disorder cases. Sleep Med. 2016, 25, 105-112. [CrossRef]

32. Fernández-de-Las-Peñas, C.; Fernández-Muñoz, J.J.; Palacios-Ceña, M.; Parás-Bravo, P.; Cigarán-Méndez, M.; Navarro-Pardo, E. Sleep disturbances in tension-type headache and migraine. Ther. Adv. Neurol. Disord. 2018, 11, 1-6. [CrossRef]

33. Popit, M.; Zaletel-Kragelj, L.; Eržen, I.; Zadravec-Zaletel, L.; Zaletel, M. Estimation of the Impact of Migraine on Self-rated Health: A Cross-sectional Study in Slovenia. Zdr. Varst. 2020, 60, 38-45. [CrossRef] [PubMed]

34. Tran, D.P.; Spierings, E.L. Headache and insomnia: Their relation reviewed. Cranio 2013, 31, 165-170. [CrossRef] [PubMed]

35. Benito-González, E.; Palacios-Ceña, M.; Fernández-Muñoz, J.J.; Castaldo, M.; Wang, K.; Catena, A.; Arendt-Nielsen, L.; Fernándezde-Las-Peñas, $C$. Variables associated with sleep quality in chronic tension-type headache: A cross-sectional and longitudinal design. PLoS ONE 2018, 13, e0197381. [CrossRef] [PubMed]

36. Rafique, N.; Al-Asoom, L.I.; Latif, R.; Alsunni, A.A.; Salem, A.M.; Alkhalifa, Z.H.; Almaharfi, R.M.; Alramadan, R.S.; Aldajani, Z.F.; Alghadeer, F.A.T.; et al. Prevalence of Migraine and its Relationship with Psychological Stress and Sleep Quality in Female University Students in Saudi Arabia. J. Pain Res. 2020, 13, 2423-2430. [CrossRef] [PubMed]

37. Chen, S.-Y.; Chen, C.-H.; Lo, C.; Lu, M.-L.; Hsu, C.-D.; Chiu, Y.-H.; Chen, C.-H.; Lin, S.-K.; Huang, M.-C.; Chen, H.-C.; et al. Differential Co-Occurring Patterns Between Depressive Symptomatology and Sleep-Wake-Related Disturbances in Mood Disorders. Nat. Sci. Sleep 2021, 13, 503-514. [CrossRef]

38. Headache Classification Committee of the International Headache Society (IHS). The International Classification of Headache Disorders, 3rd edition. Cephalalgia 2018, 38, 1-211. [CrossRef] 
39. Schiffman, E.; Ohrbach, R.; Truelove, E.; Look, J.; Anderson, G.; Goulet, J.P.; List, T.; Svensson, P.; Gonzalez, Y.; Lobbezoo, F.; et al. Diagnostic Criteria for Temporomandibular Disorders (DC/TMD) for Clinical and Research Applications: Recommendations of the International RDC/TMD Consortium Network* and Orofacial Pain Special Interest Groupdagger. J. Oral Facial Pain Headache 2014, 28, 6-27. [CrossRef]

40. Ashraf, J.; Zaproudina, N.; Suominen, A.L.; Sipilä, K.; Närhi, M.; Saxlin, T. Association Between Temporomandibular Disorders Pain and Migraine: Results of the Health 2000 Survey. J. Oral Facial Pain Headache 2019, 33, 399-407. [CrossRef]

41. Abouelhuda, A.M.; Kim, H.S.; Kim, S.Y.; Kim, Y.K. Association between headache and temporomandibular disorder. J. Korean Assoc. Oral Maxillofac. Surg. 2017, 43, 363-367. [CrossRef]

42. Slade, G.D.; Spencer, A.J. Development and evaluation of the Oral Health Impact Profile. Community Dent. Health 1994, $11,3-11$.

43. Rener-Sitar, K.; Celebic, A.; Petricevic, N.; Papic, M.; Sapundzhiev, D.; Kansky, A.; Marion, L.; Kopac, I.; Zaletel-Kragelj, L. The Slovenian version of the Oral Health Impact Profile Questionnaire (OHIP-SVN): Translation and psychometric properties. Coll. Antropol. 2009, 33, 1177-1183. [PubMed]

44. Kroenke, K.; Spitzer, R.L.; Williams, J.B.W. The PHQ-9. J. Gen. Intern. Med. 2001, 16, 606-613. [CrossRef] [PubMed]

45. Frankovič, S. Slovenian Translation and Psychometric Analysis of International Questionnaires for Assessment of Psychosocial Status of Patients with Temporomandibular Disorders. Master's Thesis, University of Ljubljana, Ljubljana, Slovenia, 2017.

46. Spitzer, R.L.; Kroenke, K.; Williams, J.B.; Löwe, B. A brief measure for assessing generalized anxiety disorder: The GAD-7. Arch Intern. Med. 2006, 166, 1092-1097. [CrossRef] [PubMed]

47. Kroenke, K.; Spitzer, R.L.; Williams, J.B. The PHQ-15: Validity of a new measure for evaluating the severity of somatic symptoms. Psychosom. Med. 2002, 64, 258-266. [CrossRef] [PubMed]

48. Ohrbach, R.; Markiewicz, M.R.; McCall Jr, W.D. Waking-state oral parafunctional behaviors: Specificity and validity as assessed by electromyography. Eur. J. Oral Sci. 2008, 116, 438-444. [CrossRef]

49. Kmetec, S.; Fekonja, Z.; Davey, A.; Mlinar Reljić, N.; Lorber, M. Development of a slovenian version of the pittsburgh sleep quality index (PSQI-SLO) for use with older adults. Int. J. Older People Nurs. 2021, 17, e12411. [CrossRef]

50. 1KA (Version 21.05.25) [Computer Program]; Faculty of Social Sciences, The University of Ljubljana: Ljubljana, Slovenia, 2017; Available online: https:/ / www.1ka.si/ (accessed on 20 January 2022).

51. Diagnostic Criteria for Temporomandibular Disorders: Scoring Manual for Self-Report Instruments. Version: March 30,2021 [Homepage on the Internet]. Buffalo: International Network for Orofacial Pain and Related Disorders Methodology. 2021. Available online: https:/ / buffalo.app.box.com/s/m97v93ct0cz08e77o2g9biu0j7asq7uk (accessed on 9 January 2022).

52. Colarusso, C.A. Young Adulthood (Ages 20-40). In Child and Adult Development: A Psychoanalytic Introduction for Clinicians; Springer: Berlin/Heidelberg, Germany, 1992; pp. 133-161.

53. Cohen, J. Statistical Power Analysis for the Behavioral Sciences; Academic Press: Cambridge, MA, USA, 2013.

54. Hosmer, D.W.; Lemeshow, S. Assessing the Fit of the Model. In Applied Logistic Regression; Shewhart, W.A., Wilks, S.S., Hosmer, D.W., Lemeshow, S., Eds.; John Wiley and Sons: New York, NY, USA, 2000; pp. 143-202.

55. Hosmer, D.W.; Lemeshow, S. Interpretation of the Fitted Logistic Regression Model. In Applied Logistic Regression; Shewhart, W.A., Wilks, S.S., Hosmer, D.W., Lemeshow, S., Eds.; John Wiley and Sons: New York, NY, USA, 2000; pp. 47-90.

56. Memmedova, F.; Emre, U.; Yalın, O.; Doğan, O.C. Evaluation of temporomandibular joint disorder in headache patients. Neurol. Sci. 2021, 42, 4503-4509. [CrossRef]

57. Fernandes, G.; Franco, A.L.; Goncalves, D.A.; Speciali, J.G.; Bigal, M.E.; Camparis, C.M. Temporomandibular disorders, sleep bruxism, and primary headaches are mutually associated. J. Orofac. Pain 2013, 27, 14-20.

58. Suvinen, T.I.; Reade, P.C.; Kemppainen, P.; Könönen, M.; Dworkin, S.F. Review of aetiological concepts of temporomandibular pain disorders: Towards a biopsychosocial model for integration of physical disorder factors with psychological and psychosocial illness impact factors. Eur. J. Pain 2005, 9, 613-633. [CrossRef]

59. Manfredini, D.; Fabbri, A.; Peretta, R.; Guarda-Nardini, L.; Lobbezoo, F. Influence of psychological symptoms on home-recorded sleep-time masticatory muscle activity in healthy subjects. J. Oral Rehabil. 2011, 38, 902-911. [CrossRef]

60. De La Torre Canales, G.; Câmara-Souza, M.B.; Muñoz Lora, V.R.M.; Guarda-Nardini, L.; Conti, P.C.R.; Rodrigues Garcia, R.M.; Del Bel Cury, A.A.; Manfredini, D. Prevalence of psychosocial impairment in temporomandibular disorder patients: A systematic review. J. Oral Rehabil. 2018, 45, 881-889. [CrossRef] [PubMed]

61. Choi, W. Effect of 4 Weeks of Cervical Deep Muscle Flexion Exercise on Headache and Sleep Disorder in Patients with Tension Headache and Forward Head Posture. Int. J. Environ. Res. Public Health 2021, 18, 3410. [CrossRef]

62. Romero-Reyes, M.; Uyanik, J.M. Orofacial pain management: Current perspectives. J. Pain Res. 2014, 7, 99-115. [CrossRef] [PubMed]

63. Reiter, S.; Emodi-Perlman, A.; Kasiel, H.; Abboud, W.; Friedman-Rubin, P.; Arias, O.W.; Manor, Y. Headache Attributed to Temporomandibular Disorders: Axis I and II Findings According to the Diagnostic Criteria for Temporomandibular Disorders. J. Oral Facial Pain Headache 2021, 35, 119-128. [CrossRef] [PubMed]

64. Tchivileva, I.E.; Ohrbach, R.; Fillingim, R.B.; Lin, F.C.; Lim, P.F.; Arbes, S.J., Jr.; Slade, G.D. Clinical, psychological, and sensory characteristics associated with headache attributed to temporomandibular disorder in people with chronic myogenous temporomandibular disorder and primary headaches. J. Headache Pain 2021, 22, 42. [CrossRef] [PubMed] 